\title{
Hybrid Formulation of the Model-Based Non-rigid Registration Problem to Improve Accuracy and Robustness
}

\author{
Olivier Clatz ${ }^{1,2}$, Hervé Delingette ${ }^{1}$, Ion-Florin Talos ${ }^{2}$, \\ Alexandra J. Golby ${ }^{2}$, Ron Kikinis ${ }^{2}$, Ferenc A. Jolesz ${ }^{2}$, \\ Nicholas Ayache ${ }^{1}$, and Simon K. Warfield ${ }^{3}$ \\ 1 Epidaure Research Project, INRIA Sophia Antipolis, France \\ 2 Surgical Planning Laboratory, Harvard Medical School, \\ Boston, USA \\ 3 Computational Radiology Laboratory, Brigham and Women's Hospital, \\ Children's Hospital, Harvard Medical School, \\ Boston, USA
}

\begin{abstract}
We present a new algorithm to register 3D pre-operative Magnetic Resonance (MR) images with intra-operative MR images of the brain. This algorithm relies on a robust estimation of the deformation from a sparse set of measured displacements. We propose a new framework to compute iteratively the displacement field starting from an approximation formulation (minimizing the sum of a regularization term and a data error term) and converging toward an interpolation formulation (least square minimization of the data error term). The robustness of the algorithm is achieved through the introduction of an outliers rejection step in this gradual registration process. We ensure the validity of the deformation by the use of a biomechanical model of the brain specific to the patient, discretized with the finite element method. The algorithm has been tested on six cases of brain tumor resection, presenting a brain shift up to $13 \mathrm{~mm}$.
\end{abstract}

\section{Introduction}

\subsection{Image-Guided Neurosurgery}

The development of intra-operative imaging systems has contributed to improving the course of intracranial neurosurgical procedures. Among these systems, the intra-operative magnetic resonance scanner offers the possibility to acquire full brain MR images in less than 4 minutes.

Intra-operative measurements show that the deformation of the brain is an important source of error that needs to be considered. Indeed, imaging the brain during the procedure makes the tumor resection more effective, and provides additional guidance for the complete resections in critical brain areas. However, even if the intra-operative MR scanner provides significantly more information than any other intra-operative imaging system, it is not clinically possible to 
acquire image modalities like diffusion tensor MR, functional MR or high resolution MR images in a reasonable time during the procedure.

Non rigid registration algorithms provide a way to overcome the intra-operative acquisition problem: instead of a time consuming acquisition of images during the procedure (dt-MRI, f-MRI or high resolution MRI), the intraoperative deformation is estimated based on fast acquisition of intra-operative images. This transformation is then applied to match the pre-operative images on the intra-operative data.

\subsection{Non-rigid Registration for Image-Guided Surgery}

Simplified biomechanical linear models have been used to interpolate the full brain deformation based on the measure of surface displacements (brain, ventricles). Audette [1] measured the visible intra-operative cortex shift using a laser range scanner. Ferrant [2] extracted the full cortex and ventricles surfaces from intra-operative MR images. These interpolation-based registration methods however suffer from a decrease of accuracy when reaching internal structures far from the measured surface.

These models are introduced through the energy minimization formulation of the registration problem as a regularization component. In 1998, Yeung [3] showed impressive registration results on a phantom using an energy minimization formulation combining ultrasound speckle tracking with a mechanical finite element model. Rohr et al. 4] combined elastic regularization with an improved block matching (BM) algorithm relying on relevant anatomical landmarks and taking into account the anisotropic matching error. In 2001, Rexilius [5] combined feature point correspondences with a finite element biomechanical model in an approximation formulation to capture brain shift.

\section{Method}

We have developed a patient-specific registration algorithm to measure the brain deformation based on two images acquired before and during the brain surgery. This algorithm can be decomposed into three main parts. The first part consists in building a biomechanical model specific to the patient corresponding to his position in the open-magnet scanner. The second part is the block (or template) matching computation for selected blocks. The third part is the new iterative hybrid solver which alternates an energy minimization step with an outlier rejection step.

In addition, we address the problem of discriminant information distribution in the images (known as the aperture problem in computer vision) to make the registration process dependent on the spatial distribution of the information given by the structure tensor (see Section 2.1 for definition).

In the following section, we propose a description of the algorithm sequence, making a distinction between off line (before the first MR acquisition to be registered) and on-line computations. 


\subsection{Pre-operative MR Image Processing}

Segmentation. We use the Brainvisa software1 to automatically segment the brain in the pre-operative images (see Figure 1, B). The tumor segmentation is manually delineated by the physician for the pre-operative planning.

Rigid Registration. An initial intra-operative MR image is acquired at the very beginning of the procedure, before opening the dura-mater. This image is used to compute the rigid transformation between the two positions of the patient in the pre-operative image and the intra-operative image.

Biomechanical Model. The patient-specific brain tetrahedral mesh is build from the previous segmentation using the GHS3D mesher [6]. The mesh generated has an average number of 1700 vertices (the surface mesh is displayed on figure 1. B), which shows to be a reasonable trade-off between the number of degrees of freedom and the number of matches.

We rely on the finite element theory and consider a quasi-incompressible linear elastic constitutive equation to characterize the mechanical behavior of the brain parenchyma: $E=694 P a$ and $\nu=0.45$. Even if CSF is incompressible, the CSF is free to flow between the ventricles and the subarachnoid space. We thus assume very soft and compressible volumes for the ventricles: $E=10 P a$ and $\nu=0.05$.

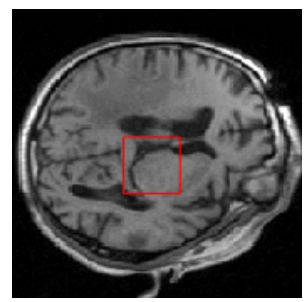

A

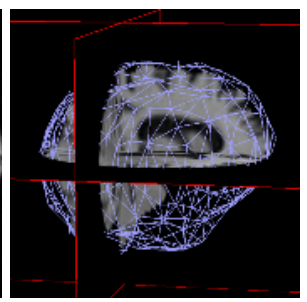

$\mathrm{B}$

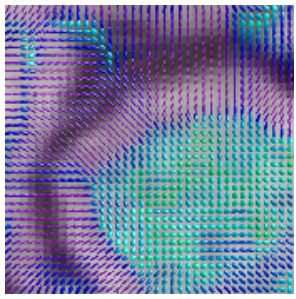

$\mathrm{C}$

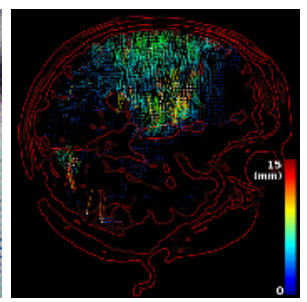

$\mathrm{D}$

Fig. 1. Illustration of the pre-operative processes. (A) pre-operative image. (B) segmentation of the brain and mesh generation (we only represent the surface mesh for visualization convenience). (C) Structure tensor visualization as ellipsoids (zoom on the square area), the color encodes the fractional anisotropy. (D) Example of a sparse displacement field computed with the block matching (BM) algorithm (5\% of the total voxels are selected as blocks centers). Color encodes displacement.

Block Selection. The relevance of a displacement estimated with a block matching (BM) algorithm depends on the presence of highly discriminant structures in this block. We use the variance of the block to measure its relevance, and only select a fraction of all potential block positions based on this criterion. In addition, we introduce the notion of prohibited connectivity between two block centers to prevent two selected blocks to be too close from each other. We obtained best results using the 26 connectivity, preventing two distinct blocks of $7 \times 7 \times 7$ voxels to share more than $42 \%$ overlapping voxels.

\footnotetext{
1 http://www.brainvisa.info/
} 
Computation of the Structure Tensor. We consider the normalized structure tensor $T_{k}$ defined in the image $I$ at position $O_{k}$ by:

$$
T_{k}=\frac{G *\left(\nabla I\left(O_{k}\right)\right)\left(\nabla I\left(O_{k}\right)\right)^{T}}{\operatorname{trace}\left[G *\left(\nabla I\left(O_{k}\right)\right)\left(\nabla I\left(O_{k}\right)\right)^{T}\right]}
$$

Where $G$ defines a convolution kernel (chosen constant in a block). Considering the classical ellipsoid representation, the more the underlying image resembles to a sharp edge, the more the structure tensor elongates in the direction orthogonal to this edge (see Figure 1, C).

\subsection{Block Matching Algorithm}

The block matching (BM) algorithm makes the assumption that a global deformation results in translation for small parts of the image: considering a block $B\left(O_{k}\right)$ in the reference image centered in $O_{k}$, and a similarity metric between two blocks $M\left(B_{a}, B_{b}\right)$, it consists in finding the positions $O^{\prime}{ }_{k}$ that maximize the similarity:

$$
\arg \max _{O_{k}^{\prime}}\left[M\left(B\left(O_{k}\right), B\left(O^{\prime}{ }_{k}\right)\right)\right]
$$

In our algorithm, the BM is performed once and only in the sgmented brain, thus restricting the displacements to the intra-cranial area (see Figure 1, D). Considering the mono-modal (MR-T1 weighted) nature of our registration problem, the correlation coefficient appears as a natural choice for the similarity measure.

\subsection{Iterative Hybrid Algorithm}

The approximation problem can be formulated as an energy minimization, composed of a mechanical and a matching (or error) energy:

$$
W_{\text {approx }}=\underbrace{U^{T} K U}_{\text {Mechanical energy }}+\underbrace{(H U-D)^{T} S(H U-D)}_{\text {Matching energy }}
$$

with:

- $U$ the displacement vector (of mesh vertices), of size $3 n$, with $\mathrm{n}$ number of vertices.

$-K$ the mesh stiffness matrix of size $3 n \times 3 n$.

$-H$ is the linear interpolation matrix in tetrahedra of size $3 p \times 3 n$.

- $D$ the block-matching computed displacement vector of size $3 p$, with p number of matched points. Note that $H U-D$ defines the estimated displacement error vector.

- $S$ is a block-diagonal matrix composed of $3 \times 3$ sub-matrices $S_{k}=\frac{\alpha}{p} c_{k} T_{k}$. The influence of a block thus depends on two factors:

1. the value of the coefficient of correlation $\left(c_{k}\right)$ : the better the correlation is (coefficient of correlation closer to 1), then the higher the influence of the block on the registration will be. 
2. The direction of matching with respect to the tensor of structure $\left(T_{k}\right)$ : we only consider the matching direction co-linear to the orientation of the intensity gradient in the block.

The $\frac{1}{p}$ factor is used to make the global matching energy independent of the number of selected blocks.

The approximation formulation however entails a systematic error: the final displacement of the brain mesh is a trade-off between the pre-operative rest position and the BM positions. An alternative approach is the interpolation formulation. The problem is turned into a mechanical energy minimization under the constraint of minimum data error, formalized with the Lagrange multipliers stored in the vector $\tilde{F}$ as:

$$
\tilde{W}_{\text {interp }}=U^{T} K U+\tilde{F}^{T} H^{T} S(H U-D)
$$

However, when some of the BM displacements are outliers, the minimization of Equation 4 may lead to unrealistic deformations.

Therefore, we propose a new iterative formulation of the registration problem:

$$
\left\{\begin{array}{l}
F_{i} \Leftarrow K U_{i} \\
U_{i+1} \Leftarrow\left[K+H^{T} S H\right]^{-1}\left[H^{T} S D+F_{i}\right]
\end{array}\right.
$$

which first solves the approximation problem (Equation 3) and gradually converges toward the interpolation solution (Equation 44). Equation 5 is iterated until the displacement modifications are smaller than a threshold. At each iteration, outliers are rejected, such that we get a more robust and unbiased estimate of the displacement. Note that $H, S$ and $D$ thus have to be recomputed at each iteration $i$.

Outlier Rejections. We introduced a robust block-rejection step based on a least-trimmed squares (LTS) estimator [7]. The LTS rejects a fraction of the total blocks based on an error function $\xi_{k}$ measuring for block $\mathrm{k}$ the error between the current mesh displacement and the matching target:

$$
\xi_{k}=\frac{\left\|S_{k}\left[(H U)_{k}-D_{k}\right]\right\|}{\lambda\left\|(H U)_{k}\right\|+1}
$$

$D_{k},(H U)_{k}$ and $\left[(H U)_{k}-D_{k}\right]$ respectively define the BM displacement, the current mesh-induced displacement and the current displacement error for block $k$. $\lambda$ is a parameter of the algorithm tailored to the error distribution on matches. With such a cost function, the rejection criterion is more flexible with points that account for larger displacements. In practice, this parameter was set to 0.5 for all our registrations. Although the least trimmed squares estimator is a robust estimator up to $50 \%$ of outliers [7], we experienced that a cumulated rejection representing $25 \%$ of the total initial selected blocks is sufficient to reject every significant outlier. The last parameter remaining in the algorithm is the matching stiffness $\alpha$. We chose a matching stiffness $\alpha=\frac{\operatorname{trace}(K)}{n}$, reflecting the average vertex stiffness (note that this value does not depend on the number of vertices used to mesh the volume), so that at least half of the displacement is already recovered after the first iteration step. 


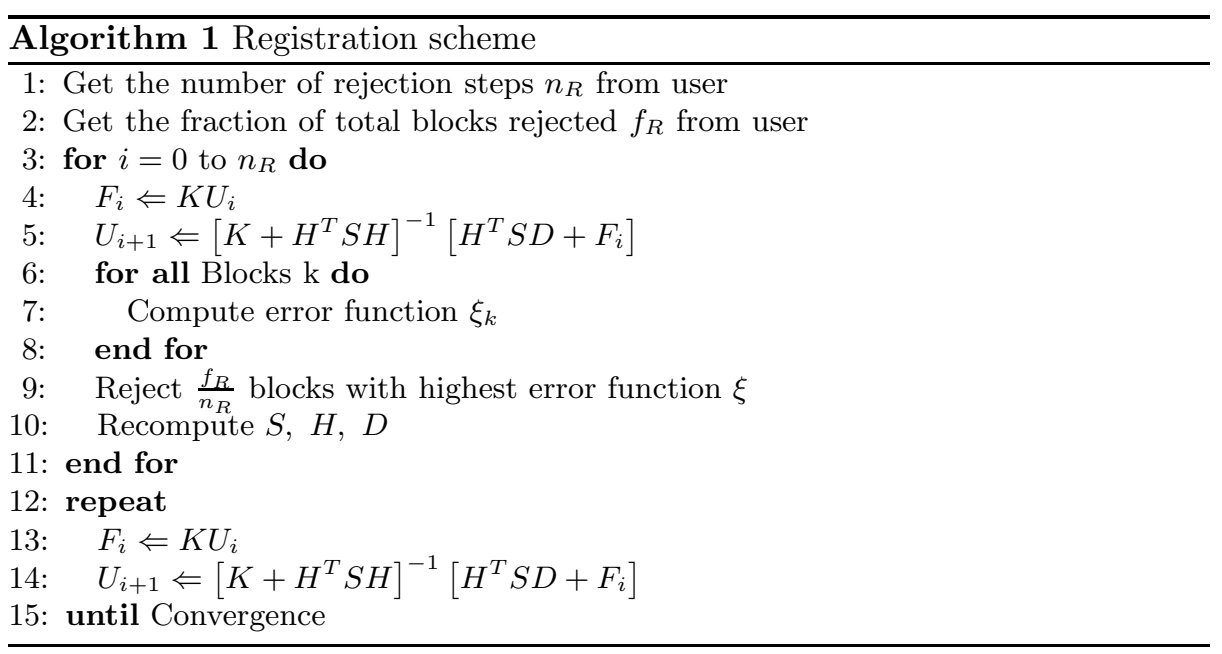

Implementation Issues and Time Constraint. We developed a parallel version of the algorithm, reducing the computation time from 162 to 25 seconds on an heterogeneous group of 15 PCs.

\section{Results}

\subsection{Experiments}

We evaluated our algorithm using the same parameters on 6 pairs of pre and intra-operative2. Figure 2 presents the results for the slice showing the largest displacement, in depth results can be seen on: http://splweb.bwh.harvard. edu:8000/pages/ppl/oclatz/registration/results.html. The quantitative accuracy of the algorithm has been evaluated by a medical expert selecting 54 corresponding feature points in the registration result image and the intraoperative image. This landmark-based error estimation has been performed on every image for 9 different points. Figure 3 shows the distribution of the landmark-based registration error as a function of the displacement of the tissue (left) and of the distance to the tumor (right). The average error on the 54 landmarks $(0.75 \mathrm{~mm})$ indicates that this algorithm is valuable for image guided therapy. The error however tends to increase in the area close to the tumor (right graph, Figure 3). We can observe that the quality of the brain segmentation has a direct influence on the deformed image, for example patient 3 of Figure 2 had a brain mask eroded on the frontal lobe which induces a missing part in the registered image. The deformation field however does not suffer from the mask inaccuracy, since the brain segmentation is not directly used to guide the registration. The assumption of local translation assumed in the block-matching

$2256 \times 256 \times 58$ slice $(0.86 \mathrm{~mm}, 0.86 \mathrm{~mm}, 2.5 \mathrm{~mm})$ acquired with the $0.5 \mathrm{~T}$ open magnet system of the Brigham and Women's hospital. 

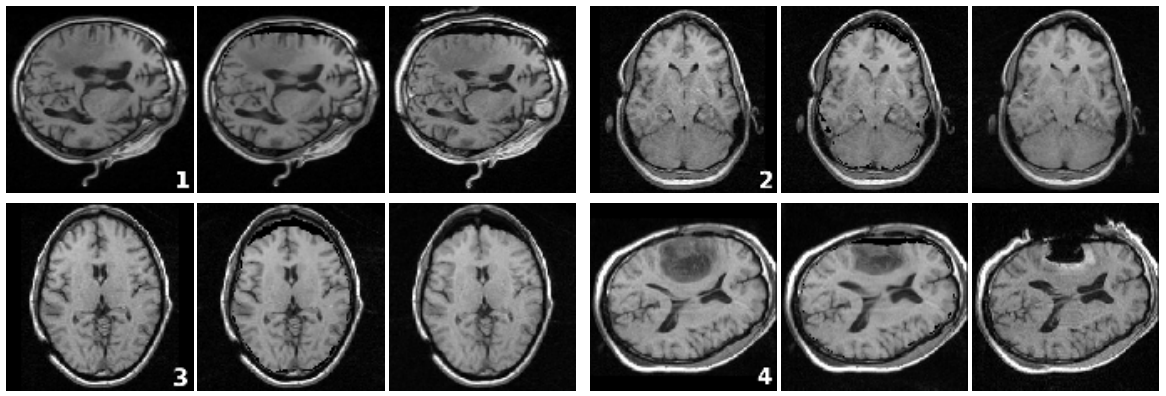

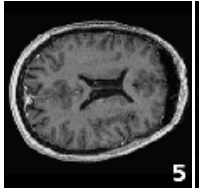

A
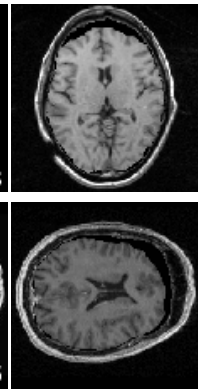

B

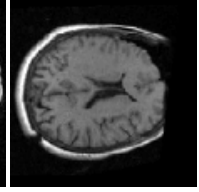

C

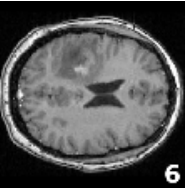

A

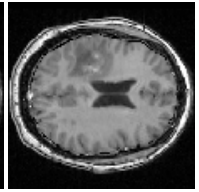

B

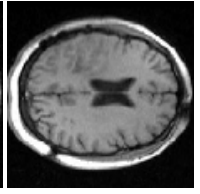

C

Fig. 2. Result of the non rigid registration of the pre-operative image on the intraoperative image for the 6 patient of our dataset. For each patient, column A shows the pre-operative image, column $\mathrm{B}$ shows the result of the registration and column $\mathrm{C}$ shows the intra-operative image (target image). The algorithm could recover large displacements (\#5), and demonstrates robustness in presence of large resection (\#4).
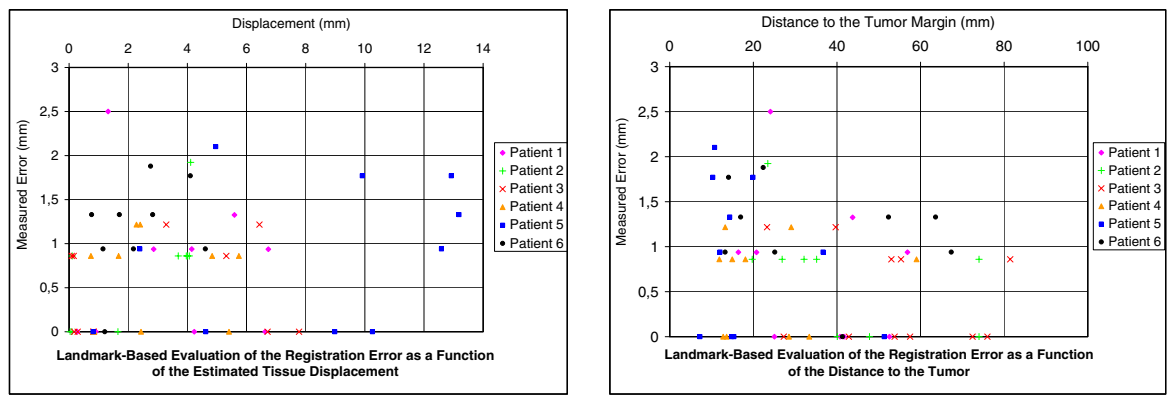

Fig. 3. Measure of the registration error for 54 landmarks. (Left) as a function of the initial arror. (Right) as a function of the distance to the tumor margin. Characteristic figures: average displacement $=3.77 \mathrm{~mm}$, maximum displacement $=13.18 \mathrm{~mm}$, average error $=0.75 \mathrm{~mm}$, maximum error $=2.50 \mathrm{~mm}$.

algorithm seems to be well adapted to the motion of the brain parenchyma. It somehow shows limitations for ventricles expansion (patient 4 and 6 of Figure 2) or collapse (patient 5 of Figure 2), where the error is approximately between two and three millimeters.

\subsection{Conclusion}

We presented in this article a new registration algorithm designed for robust nonrigid registration of intra-operative $\mathrm{MR}$ images. The algorithm has been moti- 
vated by the concept of using robust estimators to gradually move from an approximation to an interpolation formulation of the non rigid registration problem.

The results obtained with the six patients demonstrate the applicability of our algorithm to clinical cases. This method seems to be well suited to capture the mechanical brain deformation based on a sparse and noisy displacement field, limiting the error in critical regions of the brain (such as in the tumor segmentation). The remaining error may be due to the limitation of the linear elastic model.

In the future, we wish to adapt multi-scale methods to our problem, to compute near real-time deformations.

Acknowledgments. This investigation was supported by NIH grants R21 MH67054, R01 LM007861, P41 RR13218, P01 CA67165 and by a research grant from the Whitaker Foundation.

\section{References}

1. Audette, M.: Anatomical Surface Identifcation, Range-sensing and Registration for Characterizing Intrasurgical Brain Deformations. PhD thesis, McGill University (2003)

2. Ferrant, M., Nabavi, A., Macq, B., Black, P., Jolesz, F., Kikinis, R., Warfield, S.: Serial registration of intraoperative MR images of the brain. Medical Image Analysis 6 (2002) 337-360

3. Yeung, F., Levinson, S., Fu, D., Parker, K.: Feature-adaptive motion tracking of ultrasound image sequences using a deformable mesh. IEEE Transactions on Medical Imaging 17 (1998) 945-956

4. Rohr, K., Stiehl, H., Sprengel, R., Buzug, T., Weese, J., Kuhn, M.: Landmark-based elastic registration using approximating thin-plate splines. IEEE Transactions on Medical Imaging 20 (2001) 526-534

5. Rexilius, J., Warfield, S., Guttmann, C., Wei, X., Benson, R., Wolfson, L., Shenton, M., Handels, H., Kikinis1, R.: A novel nonrigid registration algorithm and applications. In: Medical Image Computing and Computer-Assisted Intervention (MICCAI'01). Volume 2208 of LNCS., Springer (2001) 923-931,

6. Frey, P.J., George, P.L.: Mesh Generation. Hermes Science Publications (2000)

7. Rousseeuw, P.: Least median-of-squares regression. Journal of the American Statistical Association 79 (1984) 871-880 